Collaborative HIV/STD Prevention Trial Group. ${ }^{1}$ UCLA, Lima, Peru: ${ }^{2}$ NAMRU-6, Callao, Peru; ${ }^{3} J o h n s$ Hopkins University, Baltimore, USA; ${ }^{4}$ UCSF, USA; ${ }^{5}$ UPCH, Lima, Peru; ${ }^{6} \mathrm{NIMH}$, USA

Objectives The NIMH Collaborative HIV/STD Intervention Trial implemented a 2-year intervention based on the theory of diffusion of innovations to decrease unprotected sex with non-primary partners among high-risk populations including heterosexually-identified, socially marginalised men in urban, coastal Peru.

Methods 20 communities in 3 cities were randomised to the intervention or comparison arm. In intervention communities, $15 \%$ of the target population was trained as Community Popular Opinion Leaders (CPOLs) to deliver HIV prevention messages to their peers. In all communities, yearly assessments of the study cohort, independent of the intervention, gathered information on sexual behaviour, provided pre-test counselling, and testing for HIV/STIs at baseline, 1 year, and 2 years after randomisation. This analysis included the 2146 heterosexually-identified men who fulfilled the trial's inclusion criteria (frequented community social venues, were aged 18 to 40, and had sex in the past 6 months).

Results Unprotected sex with a non-primary partner decreased by $10 \%$ in both study arms among heterosexually identified men. Almost half of the participants, in both the intervention and in the comparison arms, reported unprotected intercourse with a nonprimary partner at the final follow-up (47.6\% vs $48.7 \%, \mathrm{p}=0.694$ ) and there was no difference after controlling for baseline behaviour, OR 1.04, (95\% CI 0.79-to 1.38). STI incidence was also did not differ by randomisation condition, IRR 1.14 (95\% CI 0.77 - to 1.68) comparing incidence in intervention vs control communities.

Discussion We speculate that the lack of effect of the intervention among the heterosexually-identified men may be attributable to 1) an insufficiently strong intervention approach, 2) this group of men did not feel vulnerable to HIV or STI, or 3) the repeated biomedical testing and ancillary required counselling were as potent as the CPOL intervention. Future HIV prevention trials with this population should try to tailor the intervention to this group's risk perceptions and behaviours.

\section{P1-S6.46 THE LONG-TERM IMPACT OF THE MEMA KWA VIJANA ADOLESCENT SEXUAL AND REPRODUCTIVE HEALTH INTERVENTION: EFFECTS OF DOSE AND TIME SINCE EXPOSURE TO INTERVENTION}

doi:10.1136/sextrans-2011-050108.270

${ }^{1} \mathrm{~A}$ M Doyle, ${ }^{1} \mathrm{H}$ A Weiss, ${ }^{2} \mathrm{~K}$ Maganja, ${ }^{3} \mathrm{~S}$ McCormack, ${ }^{1} \mathrm{D}$ Watson-Jones, ${ }^{2} \mathrm{~J}$ Changalucha, ${ }^{1} \mathrm{R}$ J Hayes, ${ }^{1} \mathrm{D}$ A Ross. 'London School of Hygiene \& Tropical Medicine, London, UK; ${ }^{2}$ National Institute for Medical Research, Mwanza Centre, United Republic of Tanzania; ${ }^{3}$ MRC Clinical Trials Unit, UK

Background Despite recent decreases in HIV incidence in many countries in sub-Saharan Africa, there is little evidence that specific behavioural interventions have led to a reduction in HIV among young people. Further and wider-scale decreases in HIV will require better understanding of when behaviour change occurs and why. The MEMA kwa Vijana adolescent sexual and reproductive health intervention has been implemented in rural Mwanza, Tanzania since 1999. A long-term ( $9 \mathrm{yr}$ ) evaluation found that the intervention had improved knowledge, reported attitudes to sex and some sexual risk behaviours but not HIV or HSV2 prevalence. An evaluation of impact in 2001 found strong evidence that receiving more years of the intervention (dose) was associated with a larger impact on some outcomes. We explored whether there was longterm differential intervention impact according to age, marital status, dose of intervention, or time since last exposure to the inschool component of the intervention.
Methods In 2007, a cross-sectional survey was conducted in the 20 MEMA kwa Vijana trial communities among 13814 young people (15-30yrs) who had attended years 5-7 at intervention or comparison schools between 1999 and 2002. Only outcomes for which the intervention was shown to have an impact in 2001 or 2007 were included in this subgroup analysis that is, knowledge, reported attitudes, selected reported behaviours (age at first sex; number of partners; condom use). Data were analysed using clusterlevel methods for stratified cluster-randomised trials with interaction tests to determine if intervention impact differed according to subgroup.

Results Taking into account multiplicity of testing, concurrence with a priori hypotheses and consistency within the results no strong effect modifiers emerged. There was some evidence of a doseresponse effect. Specifically, impact on pregnancy knowledge increased with increasing high-quality dose of the intervention in both sexes, especially among males (Abstract P1-S6.46 table 1). There was some evidence of an impact of the high-quality dose of intervention on overall sexual attitudes among females. The consistency of these findings suggests that they are highly plausible.

Abstract P1-S6.46 Table 1 Impact of intervention in 2007/8 according to number of years of exposure to in-school intervention (1999-2002)

\begin{tabular}{|c|c|c|c|c|c|}
\hline \multirow[b]{2}{*}{ Outcome } & \multirow[b]{2}{*}{ Overall } & \multicolumn{3}{|c|}{ Years of in-school intervention ('99-'02) } & \multirow{2}{*}{$\begin{array}{l}\text { Test for } \\
\text { trend } \\
\text { (p value) }\end{array}$} \\
\hline & & $1 \mathrm{yr}$ & 2 yrs & $3+y r s$ & \\
\hline \multicolumn{6}{|c|}{ HIV acquisition knowledge (\% with all 3 responses "correct") } \\
\hline Male & $1.11(0.99,1.23)^{*}$ & 1.09 & 1.12 & 1.12 & 0.56 \\
\hline Female & $1.11(1.00,1.24)^{*}$ & 1.12 & 1.11 & 1.12 & 0.85 \\
\hline \multicolumn{6}{|c|}{ STD acquisition knowledge (\% with all 3 responses "correct") } \\
\hline Male & $1.18(1.04,1.34)^{* *}$ & 1.19 & 1.20 & 1.16 & 0.70 \\
\hline Female & $1.24(0.97,1.58)^{*}$ & 1.22 & 1.21 & 1.32 & 0.32 \\
\hline \multicolumn{6}{|c|}{ Pregnancy prevention knowledge (\% with all 3 responses "correct") } \\
\hline Male & $1.19(1.12,1.26)^{* *}$ & 1.13 & 1.19 & 1.25 & $0.0001^{* *}$ \\
\hline Female & $1.17(1.06,1.30)^{* *}$ & 1.12 & 1.20 & 1.19 & $0.03^{* *}$ \\
\hline \multicolumn{6}{|c|}{ Attitudes to sex (\% with all 3 responses "correct") } \\
\hline Male & $1.31(0.97,1.77)^{*}$ & 1.26 & 1.33 & 1.38 & 0.15 \\
\hline Female & $1.09(0.67,1.77)$ & 0.78 & 1.23 & 1.25 & $0.008^{* *}$ \\
\hline
\end{tabular}

Conclusions The desirable long-term impact of the MEMA kwa Vijana intervention did not vary greatly according to the subgroups examined. From a programme implementation point of view this suggests that the intervention can have an impact on a broad crosssection of young people in rural Mwanza.

\section{P1-S6.47 DESCRIPTION AND EVALUATION OF A REGIONAL CONDOM ACCESS PROGRAM FOR COMMUNITY ORGANIZATIONS}

doi:10.1136/sextrans-2011-050108.271

${ }^{1} \mathrm{G}$ Petit, ${ }^{2} \mathrm{~A}$ Bigras, ${ }^{2} \mathrm{C}$ Dubé, ${ }^{2} \mathrm{~F} \mathrm{C}$ Malo, ${ }^{2} \mathrm{M} \mathrm{C}$ Surprenant, ${ }^{2} \mathrm{M}$ Salvail, ${ }^{1} \mathrm{M}$ Gagnon. ${ }^{1}$ Direction de santé publique et de l'évaluation, Canada; ${ }^{2}$ Université de Sherbrooke, Sherbrooke, Canada

Background As observed in the rest of Quebec province, the number of STD declared to public health authorities in Estrie have been increasing in the last years. It has also been evaluated that condom use in this region is not as high as expected, particularly by young people. The Estrie region began a program in 2008 to favour free access to condoms for community organizations in contact with groups known as vulnerable to STDs. Purpose Describe and evaluate the implementation of the program after a period of 3 years. The regional program aim is to provide free condoms to 
vulnerable groups through distribution by community organizations in contact with these groups. This program is conducted among others to increase use of condoms and reduce STDs in Estrie. Recommendations resulting from evaluation will be use to improve the program.

Methods The process evaluation was conducted in two parts: 1 . First part was conducted by analysing all relevant documents available at the public health department in charge. 2. Second part was conducted by interviewing all community organizations which participated in the program. Information was collected orally by two interviewers, using a standardised questionnaire.

Results The regional program is based on five components: 1) Annual launch (November) with a letter sent to all community organizations known to work with vulnerable groups. 2) Project submission by community organizations using a standardised form. 3) Distribution of condoms to community organizations. 4) Distribution of condoms to vulnerable groups by community organizations. 5) Annual report of activity by participating community organizations. Eleven community organizations had received condoms within the three years of the program. More than 100000 condoms have been distributed in various projects. The regional budget allowed to the program has been able to covert the cost of all requested condoms, except for 1 year, where all projects were accepted, but with a somewhat reduced number of condoms per project. Participants were generally satisfied with the program. They confirmed its usefulness but proposed some modifications to it. Thirteen recommendations were made following evaluation. Some of them were about: 1) Favoured methods to approach community organizations. 2) Use more marketing so that more organizations know about the program. 3) Use of reminder for project submission and annual activity report. 3) Offering more kinds of condoms (especially large and without latex). 5) Offer education material to use in organizations along with condoms.

Conclusion Overall, the regional condom access program is considered useful and satisfying by the participating organizations. The recommendations resulting from evaluation will be useful to optimise the implementation of program in the future, adapt it to the needs and therefore increase its efficacy to reach its STDs reduction goals.

\section{Epidemiology poster session 6: Preventive intervention: Counselling \\ P1-S6.48 IDENTIFYING WOMEN AT RISK: CAN AN STD DIAGNOSIS HELP TARGET WOMEN FOR RISK REDUCTION COUNSELLING? MIAMI-FORT-LAUDERDALE, 2000-2009}

doi:10.1136/sextrans-2011-050108.272

${ }^{1} \mathrm{~T}$ H Brewer, ${ }^{2} \mathrm{~T}$ A Peterman, ${ }^{2} \mathrm{D}$ R Newman, ${ }^{3} \mathrm{~S}$ Shiver. ${ }^{1} \mathrm{CDC} /$ Florida DOH, Miami, USA; ${ }^{2} C D C$, Atlanta, USA; ${ }^{3}$ Florida Department of Health, Tallahassee, USA

Background The Miami-Fort Lauderdale metropolitan statistical area has one of the highest rates of HIV in the US and 29\% of cases are among women. We explored STD history as an HIV risk factor for women.

Objective To determine if reported gonorrhoea (GC), chlamydia (CT), early syphilis (ES) or demographic factors predict the risk of subsequent HIV infection among women.

Methods We linked STD surveillance data (2000-2006) and HIV report data (2000-2009) to identify HIV infection rates among females $>10$ years of age, who acquired HIV $>60$ days after an STD diagnosis. Census data were used to estimate HIV infection rates for women without an STD diagnosis. Multivariate analysis was used to identify correlates of HIV-infection in women with an STD diagnosis.

Results Of 46318 women diagnosed with an STD from 2000 to 2006, 534 (1.2\%) were subsequently reported to be HIV-infected by 2009 (median time to diagnosis 3.0 years, mean 3.5 years). Most, 446 (83.5\%), were Black. Of the 604 Black women aged 20-24 years diagnosed with HIV, 167 (27.6\%) had been diagnosed with at least one STD. However, 9187 Black women aged 20-24 had an STD in 2000-2006, so the risk of HIV following an STD diagnosis was relatively low for these women. Of 371181 Black female area residents, 6077 with no STD were reported HIV-positive in the time frame $(0.16 \%$ per person-year). Risks for Black women with STD were: CT $0.23 \%$ per person-year; GC $0.54 \%$ per person-year; and ES $1.70 \%$ per person-year. In multivariate analysis, among women with an STD, HIV risk factors were: Black race (adjusted prevalence rate ratio (PRR) 4.8, 95\% CI 3.1 to 7.3); age at STD diagnosis $<15$ years (PRR 2.1, 95\% CI 1.4 to 3.3) or between 35 and 49 years (minimal PRR 1.9, 95\% CI 1.4 to 2.5) compared to $20-24$ years; residence in a high morbidity zip code (PRR 2.1, 95\% CI 1.8 to 2.5); and diagnosis of GC (PRR 2.0, 95\% CI 1.8 to 2.5) or ES (PRR 7.9, 95\% CI 5.7 to 10.9) compared with CT.

Conclusion STDs are common in young women. However, these data demonstrate that STDs in younger and older women, particularly Blacks with gonorrhoea or syphilis are indicators of increased risk of subsequent HIV infection. These women can be easily identified in clinical settings and targeted for risk-reduction interventions.

\section{P1-S6.49 DEVELOPING AND VALIDATING A RISK SCORING TOOL FOR CHLAMYDIA INFECTION AMONG SEXUAL HEALTH CLINIC ATTENDEES IN AUSTRALIA: A SIMPLE ALGORITHM TO IDENTIFY THOSE AT HIGH RISK OF CHLAMYDIA INFECTION}

doi:10.1136/sextrans-2011-050108.273

H Wand. University of New South Wales, Sydney, Australia

Objective To develop and validate a risk scoring tool for chlamydia infection to identify those who are at increased risk of chlamydia infection.

Methods We used demographic, sexual behaviour information and chlamydia positivity results from more than 45000 individuals who attended Sydney Sexual Health Centre between 1998 and 2009. Participants were randomly allocated to either the development or internal validation dataset. Using logistic regression, we created a prediction model and weighted scoring system using the development dataset and calculated the OR of chlamydia positivity for participants in successively higher quintiles of score. The internal validation dataset was used to evaluate the performance characteristics of the model for five quintiles of risk scores including population attributable risk (PAR), sensitivity and specificity.

Results In the prediction model, inconsistent condom use, increased number of sexual partners in last 3 months, genital or anal symptoms and presenting to the clinic for sexually transmitted infections (STIs) screening or contact of an STI case were consistently associated with increased risk of chlamydia positivity in all groups. High scores (upper quintile) were significantly associated with increased risk of chlamydia infection. A cut-point score of 20 or higher distinguished a "increased risk" group with a sensitivity of 95\%, 67\% and $79 \%$ among heterosexual men, women and MSM, respectively. Conclusion The scoring tool may be included as part of a health promotion and/or clinic website to prompt those who are at increased risk of chlamydia infection which may potentially lead to increased uptake and frequency of testing. 